\title{
Induced $I_{\infty}$ stability of fixed-point digital filters without overflow oscillations and instability due to finite word length effects
}

\author{
Choon Ki Ahn ${ }^{*}$ and Young Sam Lee ${ }^{2}$
}

\author{
* Correspondence: hironaka@snut. \\ ac.kr \\ ${ }^{1}$ Department of Mechanical \& \\ Automotive Engineering, Seoul \\ National University of Science \& \\ Technology, 172 Gongneung 2- \\ dong, Nowon-gu, Seoul 139-743, \\ Korea \\ Full list of author information is \\ available at the end of the article
}

\begin{abstract}
This article studies a new criterion for the induced $I_{\infty}$ stability of fixed-point statespace digital filters without overflow oscillations and instability due to finite word length effects. The criterion not only guarantees exponential stability but also reduces the effect of external interference to an induced / norm constraint. We present a numerical example, which demonstrates the effectiveness of the proposed criterion.
\end{abstract}

\section{Introduction}

When designing a linear time-invariant digital filter using a fixed-point arithmetic, one encounters quantization and overflow nonlinearities. The presence of these nonlinearities may result in the instability of designed filters. The zero-input limit cycles, which are undesirable, may possibly occur due to these nonlinearities. The quantization and overflow nonlinear-ities may interact with each other. However, if the number of quantization steps is large, quantization effects may be decoupled when investigating the effects of overflow. Several researchers have studies stability criteria for digital filters employing saturation overflow arithmetic [1-8].

In the hardware implementation of high-order digital filters, they are usually split into some biquad filters before hardware implementation. Then, there may exist interferences between these biquad filters. These interferences lead to malfunction as well as destruction in the last $[9,10]$. However, most existing stability criteria for digital filters are only available under specific conditions without external interference, while in unfavorable environments with external interference, unfortunately, we cannot use these existing stability criteria any more. Therefore, it is important to study an alternative criterion that can overcome the shortcomings of existing stability criteria for digital filters.

There always exist model uncertainties and external disturbances in real physical systems. In recent years, this had led to an interest in the induced $l_{\infty}$ approach $[11,12]$. The induced $l_{\infty}$ approach is an effective tool to treat several dynamic systems because we can obtain general stability results using only inputs and outputs measurements. Now, the following question arises: is there an induced $l_{\infty}$ stability condition for digital filters with external interference and saturation arithmetic? However, as far as we are 
aware, for the induced $l_{\infty}$ stability for digital filters with external interference and saturation arithmetic, there are no results published in the literature so far.

This article studies a new stability criterion for fixed-point state-space digital filters with external interference and saturation arithmetic via the induced $l_{\infty}$ stability approach. This criterion is a new contribution in fields of digital filters. Under the proposed criterion, the digital filter is exponentially stable and the induced $l_{\infty}$ norm from the external interference to the state vector is reduced to an interference attenuation level. For a fixed scalar variable, we represent this criterion in terms of linear matrix inequalities (LMIs), which can be solved efficiently via existing numerical algorithms such as interior point algorithms [13,14].

This article is organized as follows. In Section 2, a new criterion for the induced $l_{\infty}$ stability of fixed-point state-space digital filters is proposed. In Section 3, a numerical example is given, and finally, conclusions are presented in Section 4.

\section{New induced $I_{\infty}$ stability criterion}

The digital filter under consideration is described by:

$$
\begin{aligned}
& x(r+1)=f(\gamma(r))+w(r) \\
& =\left[f_{1}\left(\gamma_{1}(r)\right) f_{2}\left(\gamma_{2}(r)\right) \cdots f_{n}\left(\gamma_{n}(r)\right)\right]^{T}+\left[w_{1}(r) w_{2}(r) \cdots w_{n}(r)\right]^{T}, \\
& \gamma(r)=\left[\gamma_{1}(r) y_{2}(r) \cdots y_{n}(r)\right]^{T} \\
& =A x(r) \text {, } \\
& z(r)=\left[z_{1}(r) z_{2}(r) \cdots z_{p}(r)\right]^{T} \\
& =H x(r) \text {, }
\end{aligned}
$$

where $x(r) \in R^{n}$ is the state vector, $z(r) \in R^{p}$ is a linear combination of the states, $w$ $(r) \in R^{n}$ is the external interference, $A \in R^{n \times n}$ is the coefficient matrix, and $H \in R^{p \times n}$ is a known constant matrix. The following saturation nonlinearities:

$$
f_{i}\left(y_{i}(r)\right)= \begin{cases}1, & \text { if } y_{i}(r)>1 \\ y_{i}(r), & \text { if }-1 \leq y_{i}(r) \leq 1 \\ -1, & \text { if } y_{i}(r)<-1\end{cases}
$$

are under consideration for $i=1,2, \ldots, n$. Note that the saturation nonlinearities are confined to the sector $[0,1]$, i.e.,

$$
f_{i}(0)=0, \quad 0 \leq \frac{f_{i}\left(y_{i}(r)\right)}{y_{i}(r)} \leq 1, \quad i=1,2, \ldots, n .
$$

In this article, given a level $\gamma>0$, we find a new induced $l_{\infty}$ stability criterion such that the digital filter (1)-(3) with $w(r)=0$ is exponentially stable and

$$
\sup _{r \geq 0}\left\{z^{T}(r) z(r)\right\}<\gamma^{2} \sup _{r \geq 0}\left\{w^{T}(r) w(r)\right\}
$$

under zero-initial conditions for all nonzero $w(r)$. The parameter $\gamma$ is called the induced $l_{\infty}$ norm bound or the interference attenuation level. In this case, the digital filter (1)-(3) is said to be exponentially stable with induced $l_{\infty}$ performance $\gamma$.

In the following theorem, we present a new induced $l_{\infty}$ stability criterion for digital filters. 
Theorem 1. For a given level $\gamma>0$, if we assume that there exist a symmetric positive definite matrix $P$, a positive definite diagonal matrix $M$, positive scalars $\delta, \lambda$, and $\mu$ such that

$$
\begin{aligned}
& {\left[\begin{array}{ccc}
\delta A^{T} A-P-\lambda P & M A & 0 \\
A^{T} M & P-\delta I-2 M & P \\
0 & P & P-\gamma I
\end{array}\right]<0,} \\
& {\left[\begin{array}{ccc}
\lambda P & 0 & H^{T} \\
0 & (\gamma-\mu) I & 0 \\
H & 0 & \gamma I
\end{array}\right]>0,}
\end{aligned}
$$

then the digital filter (1)-(3) is exponentially stable with induced $l_{\infty}$ performance $\gamma$.

Proof. Consider the following Lyapunov function: $V(x(r))=x^{T}(r) P x(r)$. Along the trajectory of the digital filter (1), we have

$$
\begin{aligned}
\Delta V(x(r))= & V(x(r+1))-V(x(r)) \\
= & {[f(A x(r))+w(r)]^{T} P[f(A x(r))+w(r)]-x^{T}(r) P x(r) } \\
= & f^{T}(A x(r)) P f(A x(r))+f^{T}(A x(r)) P w(r)+w^{T}(r) P f(A x(r))+w^{T}(r) P w(r) \\
& -x^{T}(r) P x(r)+2 f^{T}(A x(r)) M[A x(r)-f(A x(r))]-2 f^{T}(y(r)) M[y(r)-f(y(r))] .
\end{aligned}
$$

From (5), it is clear that

$$
f^{T}(A x(r)) f(A x(r))=\|f(A x(r))\|^{2} \leq\|A x(r)\|^{2}=(A x(r))^{T} A x(r) .
$$

Then, for a positive scalar $\delta$, we have

$$
\delta\left[x^{T}(r) A^{T} A x(r)-f^{T}(A x(r)) f(A x(r))\right] \geq 0 .
$$

If we use (10), we obtain a new upper bound for $\Delta V(x(r))$ as

$$
\begin{aligned}
\Delta V(x(r)) \leq & f^{T}(A x(r)) P f(A x(r))+f^{T}(A x(r)) P w(r)+w^{T}(r) P f(A x(r))+w^{T}(r) P w(r) \\
& -x^{T}(r) P x(r)+2 f^{T}(A x(r)) M[A x(r)-f(A x(r))]-2 f^{T}(y(r)) M[\gamma(r)-f(y(r))] \\
& +\delta\left[x^{T}(r) A^{T} A x(r)-f^{T}(A x(r)) f(A x(r))\right] \\
= & {\left[\begin{array}{c}
x(r) \\
f(A x(r)) \\
w(r)
\end{array}\right]^{T}\left[\begin{array}{ccc}
\delta A^{T} A-P+\lambda P & M A & 0 \\
A^{T} M & P-\delta I-2 M & P \\
0 & P & P-\mu I
\end{array}\right]\left[\begin{array}{c}
x(r) \\
f(A x(r)) \\
w(r)
\end{array}\right] } \\
& -\lambda x^{T}(r) P x(r)+\mu w^{T}(r) w(r)+\Phi(r),
\end{aligned}
$$

where $\Phi(r)=-2 f^{T}(y(r)) M[y(r)-f(y(r))]$. Note that $\Phi(r)$ is nonpositive in view of (4). If the matrix inequality (7) is satisfied, we have

$$
\begin{aligned}
\Delta V(x(r)) & <-\lambda x^{T}(r) P x(r)+\mu w^{T}(r) w(r) \\
& =-\lambda V(x(r))+\mu w^{T}(r) w(r) .
\end{aligned}
$$

Hence $\Delta V(x(r))<0$ holds, whenever $V(x(r)) \geq \frac{\mu}{\lambda} w^{T}(r) w(r)$. Since $V(x(0))=0$ under the zero-initial condition, this shows that $V(x(r))$ cannot exceed the value $\frac{\mu}{\lambda} w^{T}(r) w(r)$

$$
x^{T}(r) P x(r)=V(x(r))<\frac{\mu}{\lambda} w^{T}(r) w(r)
$$


for $r \geq 0$. It follows from (13) that

$$
\begin{aligned}
\frac{1}{\gamma} & x^{T}(r) H^{T} H x(r)-\gamma w^{T}(r) w(r) \\
& =\frac{1}{\gamma} x^{T}(r) H^{T} H x(r)-(\gamma-\mu) w^{T}(r) w(r)-\mu w^{T}(r) w(r) \\
& <\frac{1}{\gamma} x^{T}(r) H^{T} H x(r)-(\gamma-\mu) w^{T}(r) w(r)-\lambda x^{T}(r) \operatorname{Px}(r) .
\end{aligned}
$$

The matrix inequality (8) gives

$$
\frac{1}{\gamma}\left[\begin{array}{c}
H^{T} \\
0
\end{array}\right][H 0]<\left[\begin{array}{cc}
\lambda P & 0 \\
0 & (\gamma-\mu) I
\end{array}\right] .
$$

Pre- and post-multiplying (15) by $\left[x^{T}(r) w^{T}(r)\right]$ and $\left[x^{T}(r) w^{T}(r)\right]^{T}$, respectively, yields

$$
\frac{1}{\gamma} x^{T}(r) H^{T} H x(r)-(\gamma-\mu) w^{T}(r) w(r)-\lambda x^{T}(r) P x(r)<0,
$$

which ensures

$$
\frac{1}{\gamma} x^{T}(r) H^{T} H x(r)-\gamma w^{T}(r) w(r)<0
$$

from (14). Thus, we have

$$
\begin{aligned}
z^{T}(r) z(r) & =x^{T}(r) H^{T} H x(r) \\
& <\gamma^{2} w^{T}(r) w(r) .
\end{aligned}
$$

Taking the supremum over $r \geq 0$ leads to (6).

Next, we show that, under the conditions (7) and (8), the filter (1)-(3) with $w(r)=0$ is exponentially stable. $V(x(r))$ satisfies the following Rayleigh inequality [15]:

$$
\lambda_{\min }(P)\|x(r)\|^{2} \leq V(x(r)) \leq \lambda_{\max }(P)\|x(r)\|^{2},
$$

where $\lambda_{\max }(\cdot)$ and $\lambda_{\min }(\cdot)$ are the maximum and minimum eigenvalues of the matrix. When $w(r)=0$, we have

$$
\Delta V(x(r))<-\lambda V(x(r))=-\lambda x^{T}(r) P x(r) \leq-\lambda \min (P)\|x(r)\|^{2}
$$

from (12). According to Theorem 3.1 of [16], (19) and (20) guarantee the exponential stability of the digital filter (1)-(3). This completes the proof.

Corollary 1. If $w(r)$ is bounded as $w^{T}(r) w(r)<\chi$, the conditions (7) and (8) guarantee that $x(r)$ is bounded as

$$
\|x(r)\|<\sqrt{\frac{\mu \chi}{\lambda_{\min }(P) \lambda}}, \quad r \geq 0 .
$$

Proof. From (13), we have

$$
\lambda_{\min }(P)\|x(r)\|^{2} \leq x^{T}(r) P x(r)=V(x(r))<\frac{\mu}{\lambda} \chi .
$$

Thus, we obtain the relation (21). This completes the proof.

Remark 1. Recently, an $\mathcal{H}_{\infty}$ (or energy-to-energy) stability criterion for fixed-point state-space digital filters with saturation arithmetic and external interference was 
proposed in [17]. In contrast to this study, the induced $l_{\infty}$ stability criterion can handle the worst-case peak value of the state vector for all bounded peak values of the disturbance signals.

Remark 2. For a fixed positive scalar $\lambda$, (7) and (8) are LMIs. We can apply various convex optimization algorithms to check whether these LMIs are feasible. In order to solve these LMIs, this article used MATLAB LMI Control Toolbox [14].

Remark 3. The $l_{\infty}$ induced norm [11,12] is defined as

$$
\left\|T_{z w}\right\|_{l_{\infty}}=\frac{\sqrt{\sup _{r \geq 0}\left\{z^{T}(r) z(r)\right\}}}{\sqrt{\sup _{r \geq 0}\left\{w^{T}(r) w(r)\right\}}}
$$

where $T_{z w}$ is a transfer function matrix from $w(r)$ to $z(r)$. For a given level $\gamma>0,\left\|T_{z w}\right\|_{l_{\infty}}<\gamma$ can be restated in the equivalent form (6). If we define

$$
L(r)=\frac{\sup _{0 \leq k \leq r}\left\{z^{T}(k) z(k)\right\}}{\sup _{0 \leq k \leq r}\left\{w^{T}(k) w(k)\right\}},
$$

the relation (6) can be represented by $L(\infty)<\gamma^{2}$. In the following section, through the plot of $L(r), L(\infty)<\gamma^{2}$ is verified.

\section{Numerical example}

Consider a second-order filter (1)-(3) with

$$
A=\left[\begin{array}{cc}
0.25 & 0.5 \\
-0.5 & 0.8
\end{array}\right], \quad H=\left[\begin{array}{cc}
0.1 & 0 \\
0 & 0.1
\end{array}\right], \quad w(r)=0.5\left[\begin{array}{c}
\cos (2 r) \\
2 \sin (r)
\end{array}\right] .
$$

Let the induced $l_{\infty}$ performance be specified by $\gamma=0.3$. In addition, we fix $\lambda=1$. Solving (7) and (8) by the convex optimization technique of MATLAB software gives

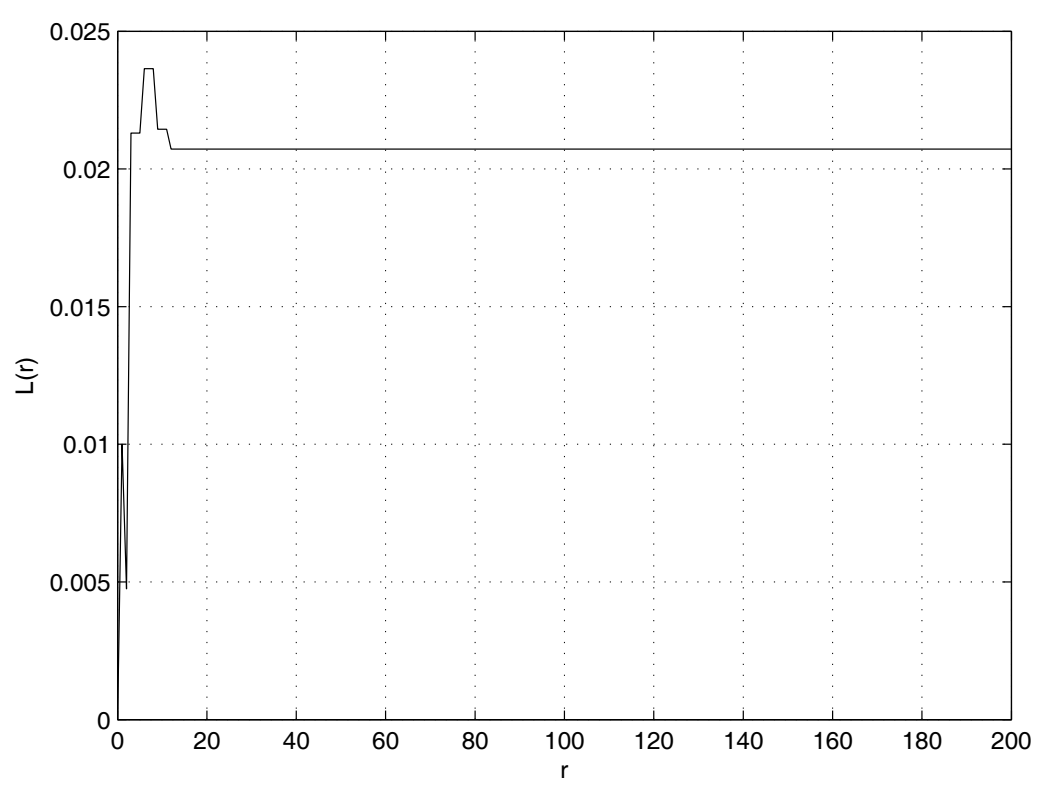

Figure 1 The plot of $L(r)$ 


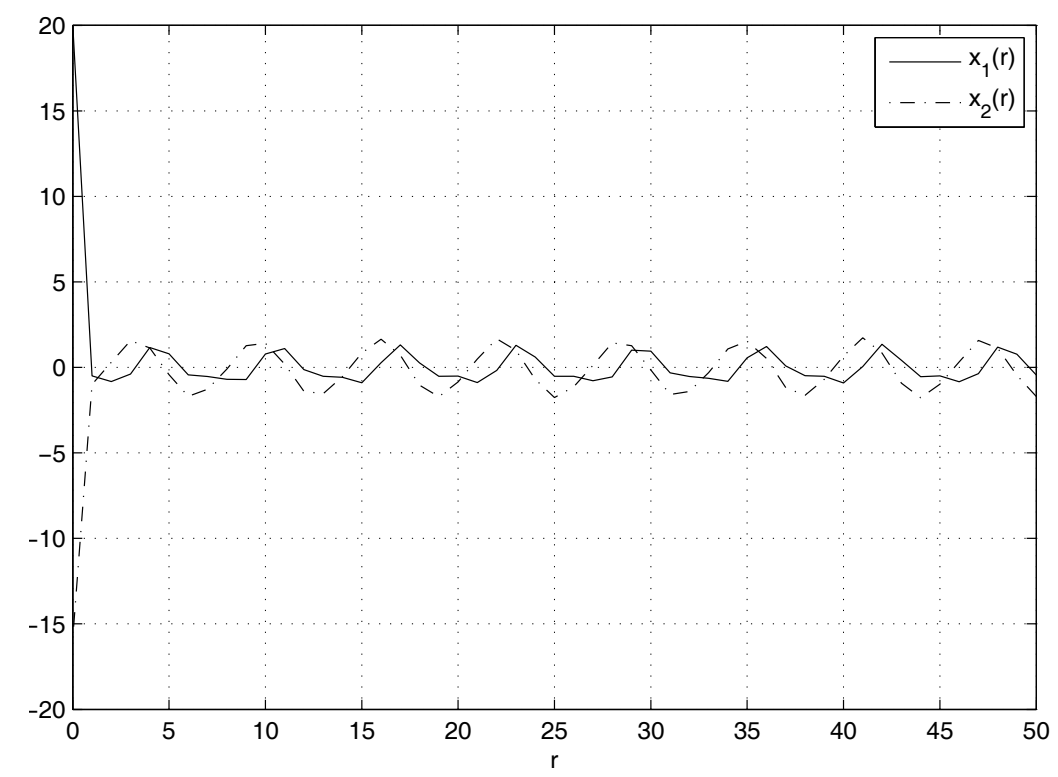

Figure 2 State trajectories

Figure 1 shows the plot of $L(r)$, which is defined in (23). This figure verifies $L(\infty)<\gamma^{2}=0.09$, which means that the induced $l_{\infty}$ norm from $w(r)$ to $z(r)$ is reduced within the induced $l_{\infty}$ norm bound $\gamma$. Figure 2 represents the state trajectories of the digital filter (1)-(3) when $\left(x_{1}\right.$ $\left.(0), x_{2}(0)\right)=(20,-15.8)$. It is clear that stability criteria in existing studies [1-8] fail in the filter given by (1)-(3) with the parameters (24). On the other hand, the proposed criterion (7) and (8) verifies the exponential stability result with induced $l_{\infty}$ performance in this example.

\section{Conclusion}

This article studies a new criterion for the induced $l_{\infty}$ stability of fixed-point statespace digital filters with external interference and saturation overflow arithmetic. It is shown that the criterion can ensure to reduce the effect of the external interference to a prescribed attenuation level. Thus, it can overcome the disadvantages of existing stability criteria. For a fixed scalar variable, this criterion is represented in terms of LMIs and, hence, computationally tractable. Finally, a numerical example shows the usefulness of the proposed stability criterion.

Acknowledgements

This research was supported by the MKE (The Ministry of Knowledge Economy), Korea, under the CITRC (Convergence Information Technology Research Center) support program (NIPA-2011-C6150-1102-0001) supervised by the NIPA (National IT Industry Promotion Agency).

\section{Author details}

'Department of Mechanical \& Automotive Engineering, Seoul National University of Science \& Technology, 172 Gongneung 2-dong, Nowon-gu, Seoul 139-743, Korea 2Department of Electrical Engineering, Inha University, 253 Younghyun-dong, Nam-gu, Incheon 402-751, Korea

Authors' contributions

All authors contributed equally and significantly in writing this article. All authors read and approved the final manuscript. 


\section{Competing interests}

The authors declare that they have no competing interests.

Received: 1 March 2012 Accepted: 23 April 2012 Published: 23 April 2012

\section{References}

1. Ebert, P, Mazo, J, Taylor, M: Overflow oscillations in recursive digital filters. Bell Syst Tech J. 48, 2999-3020 (1969)

2. Sandberg, I: The zero-input response of digital filters using saturation arithmetic. IEEE Trans Circ Syst. 26, 911-915 (1979). doi:10.1109/TCS.1979.1084587

3. Singh, V: Elimination of overflow oscillations in fixed-point state-space digital filters using saturation arithmetic. IEEE Trans Circ Syst. 37, 814-818 (1990). doi:10.1109/31.55040

4. Kar, $\mathrm{H}$, Singh, $\mathrm{V}$ : A new criterion for the overflow stability of second-order state-space digital filters using saturation arithmetic. IEEE Trans Circ Syst I. 45, 311-313 (1998). doi:10.1109/81.662720

5. Kar, H, Singh, V: Stability analysis of discrete-time systems in a state-space realisation with partial state saturation nonlinearities. IEE Proc Control Theory Appl. 150, 205-208 (2003). doi:10.1049/ip-cta:20030208

6. Kar, H, Singh, V: Elimination of overflow oscillations in digital filters employing saturation arithmetic. Digital Signal Process. 15, 536-544 (2005). doi:10.1016/j.dsp.2005.02.001

7. Singh, V: LMI approach to stability of direct form digital filters utilizing single saturation overflow nonlinearity. IEEE Trans Circ Syst II. 54, 878-881 (2007)

8. Singh, V: Stability analysis of a class of digital filters utilizing single saturation nonlin-earity. Automatica. 44, 282-285 (2008). doi:10.1016/j.automatica.2007.04.015

9. Tsividis, Y: Mixed Analog-Digital V LSI Devices and Technology. World Scientific Publishing, Singapore (2002)

10. Monteiro, J, Leuken, R: Integrated Circuit and System Design: Power and Timing Modeling, Optimization and Simulation. Springer, New York (2010)

11. Abedor, J, Nagpal, K, Poolla, K: A linear matrix inequality approach to peak-to-peak gain minimization. Int J Robust Nonlinear Control. 6, 899-927 (1996). doi:10.1002/(SICl)1099-1239(199611)6:9/103.0.CO;2-G

12. Scherer, C, Gahinet, P, Chilali, M: Multiobjective output-feedback control via LMI optimization. IEEE Trans Automat Control. 42, 896-911 (1997). doi:10.1109/9.599969

13. Boyd, S, Ghaoui, LE, Feron, E, Balakrishinan, V: Linear Matrix Inequalities in Systems and Control Theory. SIAM, Philadelphia, PA (1994)

14. Gahinet, P, Nemirovski, A, Laub, AJ, Chilali, M: LMI Control Toolbox. The Mathworks Inc., Natick, MA (1995)

15. Strang, G: Introduction to Applied Mathematics. Wellesley Cambridge Press, Cambridge (1986)

16. Lee, J: Constructive and discrete versions of the Lyapunov's stability theorem and the LaSalle's invariance theorem. Commun Korean Math Soc. 17, 155-163 (2002)

17. Ahn, C: Criterion for the overflow stability of fixed-point digital filters with saturation arithmetic and external interference. Int J Electron Commun. 65(9):750-752 (2011). doi:10.1016/j.aeue.2010.12.001

doi:10.1186/1687-1847-2012-51

Cite this article as: Ahn and Lee: Induced $l_{\infty}$ stability of fixed-point digital filters without overflow oscillations and instability due to finite word length effects. Advances in Difference Equations 2012 2012:51.

\section{Submit your manuscript to a SpringerOpen ${ }^{\circ}$ journal and benefit from:}

- Convenient online submission

- Rigorous peer review

- Immediate publication on acceptance

- Open access: articles freely available online

- High visibility within the field

- Retaining the copyright to your article

Submit your next manuscript at $\boldsymbol{\wedge}$ springeropen.com 